\title{
Caractérisation biologique de différents isolats du virus de la mosaïque du chou fleur (CaMV). 1. Plantes hôtes : Solanées
}

\author{
S Mevel, C Kerlan \\ INRA, station de pathologie végétale, 35650 Rennes-le-Rheu; France
}

(Reçu le 11 décembre 1989; accepté le 9 septembre 1990)

\begin{abstract}
Résumé - L'aptitude du CaMV à infecter une gamme de Solanées a été étudiée à partir de 11 isolats prélevés sur chou-fleur en Bretagne, 2 souches provenant de Crucifères en Argentine et 5 souches de référence, Cabb-S, Cabb-B, Cabb-BJI, PV-45, CM4-184. Six isolats français, plus 2 souches argentines et Cabb-BJI sont systémiques sur Datura stramonium. Sur Nicotiana bigelowi, un isolat français et une souche argentine sont systémiques, alors que sur $N$ clevelandii, seules les 2 souches argentines le sont. $N$ benthamiana est une nouvelle plante hôte à infection systémique pour le CaMV au vu des réactions induites par 3 isolats français, les 2 souches argentines et Cabb-BJl. $N$ edwardsonii, $\mathbf{N}$ glutinosa, $\boldsymbol{N}$ glauca, $\boldsymbol{N}$ tabacum var Turkish, Samsum et Xanthi et $\mathbf{N}$ rustica ne présentent à l'infection que des symptômes locaux. Tous les isolats expérimentés sont capables d'infecter au moins 1 de ces plantes hôtes, mais aucune de celles-ci n'est sensible à tous les isolats. En raison de la grande diversité des symptômes qu'il exprime, notamment de ceux qui apparaissent pour certains isolats sur fruits et graines, $D$ stramonium constitue un hôte différenciateur particulièrement intéressant. Une seule espèce, $N$ debneyi s'est révélée immune, quels que soient la souche ou l'isolat. Enfin, cette étude montre que les isolats naturels de CaMV capables d'infecter systémiquement des Solanées sont, au moins dans certaines régions de culture du chou-fleur, plus répandus que ne le laissaient prévoir les études antérieures.
\end{abstract}

caulimovirus / test ELISA / transmission mécanique

Summary - Biological properties of different isolates of cauliflower mosaic virus (CaMV). 1. Solanaceous hosts. The behaviour of CaMV of various origins, mechanically transmitted to a range of Solanaceous plants, was studied. Eleven CaMV isolates from cauliflower cultivated in France and 2 strains from Argentina were compared with 5 standard strains, Cabb-S, Cabb-B, Cabb-BJI, PV-45, CM4-184. Six French isolates, and both strains from Argentina and Cabb-BJl, systemically infected Datura stramonium, induced various symptoms on the leaves: chlorotic and/or necrotic spots, sometimes associated with top necrosis (table II, figs 1-5). Systemic reactions also appeared on 3 Nicotiana species (table III). N bigelowii, inoculated by 1 French isolate and 1 strain from Argentina, displayed necrotic spots on non-inoculated leaves. Systemic necrotic spots were also observed on $\mathrm{N}$ clevelandii infected with the 2 strains from Argentina. $\mathrm{N}$ benthamiana was recorded for the first time as a CaMV systemic host-plant as shown by the vein-clearing and/or chlorotic spots induced by 3 French isolates, both strains from Argentina and Cabb-BJI (table III, figs 6 and 7). In our experimental conditions, $\mathrm{N}$ edwardsonii, $\mathrm{N}$ glutinosa, $\mathrm{N}$ glauca, $\mathrm{N}$ tabacum (cvs Turkish, Samsum and Xanthi) and $\mathrm{N}$ rustica only showed chlorotic or necrotic local lesions. All tested strains were able to infect at least one host species, but none of these were susceptible to all strains. Because of the large range of symptoms occurring on leaves, fruits and seeds of D stramonium, this latter appeared as a particularly convenient host-plant to discriminate between the isolates. Only 1 species, $\mathrm{N}$ debneyi was immune to all tested strains and isolates. This study showed that natural CaMV isolates able to systematically infect Solanaceous plants could be more frequent than commonly accepted, at least in some districts of cauliflower crops.

caulimovirus / ELISA / mechanical transmission 


\section{INTRODUCTION}

Lors d'une prospection réalisée en culture de chou-fleur en Bretagne, nous avions mis en évidence de nombreux isolats du virus de la Mosaïque du chou-fleur (CaMV) (Shepherd, 1981) provoquant une réaction d'hypersensibilité sur Nicotiana clevelandii Gray (Kerlan et Mevel, 1989). Les souches de ce virus capables d'infecter des plantes autres que des Crucifères sont cependant peu fréquences; la première a été découverte par Hills et Campbell (1968) associée au Broccoli Necrotic Yellows Virus chez des choux-broccolis; Lung et Pirone (1972) ont ensuite montré que Datura stramonium $\mathrm{L}$ est un hôte à lésions locales pour 4 isolats de CaMV, parmi lesquels Cabb-B isolé à partir de Brassica aux USA (Walker et al, 1945). Au cours des dernières années, de nouvelles souches - ou isolats - systémiques sur Solanées, ont été décrites; W 260, W 262 et W 283, proviennent de prélèvements effectués sur Crucifères dans la région de Mendoza en Argentine (Garcia et Shepherd, 1985); une autre souche de ce type, D4, dérive d'un inoculum (Fordham isolate) isolé sur navet à Davis aux USA (Schoelz et al, 1986).

Le comportement de 2 de ces souches, $W$ 260 et D4, sur une gamme restreinte de Solanées a fait l'objet d'une étude approfondie, en relation avec leurs caractéristiques génomiques; la comparaison des séquences nucléotidiques des souches Solanées avec celles de souches inféodées aux Crucifères ou celles de recombinants artificiels, semble permettre de déterminer les gènes ou fragments de gènes impliqués dans la spécificité vis-à-vis de l'hôte, le type d'infection et l'expression de la symptomatologie (Schoelz et Shepherd, 1988). En raison de l'intérêt scienti- fique actuel pour les souches particulières de CaMV, il nous a paru intéressant de parvenir à une meilleure caractérisation des isolats récoltés en Bretagne et de réaliser en premier lieu une étude complémentaire de leurs plantes hôtes; le présent article décrit les résultats obtenus sur une gamme élargie de Solanées.

\section{MATÉRIEL. ET MÉTHODES}

\section{Matériel}

\section{Virus}

Dix-huit isolats et souches de CaMV ont été expérimentés. Onze isolats proviennent de prélèvements effectués sur chou-fleur en Bretagne et représentent des groupes d'isolats de CaMV définis antérieurement; ce sont les isolats B-7, B-12, B-17, B-20, B-21, B-22, B-23, B-26, B-29, B-30, B-32 (Kerlan et Mevel, 1989). Cinq souches Cabb-S, Cabb-B, Cabb-BJI, PV45 et CM4-184, précédemment utilisées dans le travail cité ci-dessus, ont été conservées comme matériel de référence. Deux souches originaires d'Argentine, W 260 et $A 517$ ont également été étudiées (tableau I).

Ces 11 isolats et ces 7 souches ont été maintenues par inoculation mécanique sur Brassica campestris $\mathrm{L}$ en serre hermétique aux pucerons dans des conditions déjà décrites (Kerlan et Mevel, 1989).

\section{Plantes tests}

Douze espèces et variétés de plantes tests ont été exprimentées : Duatra stramonium L, Nicotiana bigelowii $L, N$ clevelandii Gray, $N$ benthamiana Domin, $N$ edwardsonii Christie et Hall, $N$ glutinosa L, $N$ glauca Graham, $N$ tabacum $L$ Turkish, $N$ tabacum $L$ Samsum, $N$ tabacum L Xanthi, $N$ rustica $L, N$ debneyi Domin.

Tableau l. Origine des souches étrangères de CaMV utilisées.

\begin{tabular}{|c|c|c|c|c|}
\hline Souche & $\begin{array}{l}\text { Plante naturellement } \\
\text { infectée }\end{array}$ & Lieu de prélèvement & Obtenteur & Référence \\
\hline $\begin{array}{l}\text { Cabb-S } \\
\text { Cabb-B } \\
\text { Cabb-BJI } \\
\text { PV-45 ATCC } \\
\text { CM4-184 } \\
\text { W } 260 \\
\text { A } 517\end{array}$ & $\begin{array}{l}\text { Crucifères } \\
\text { chou } \\
\text { dérivé de Cabb-B } \\
\text { chou } \\
\text { Brassica sp } \\
\text { Crucifères } \\
\text { Crucifères }\end{array}$ & $\begin{array}{l}\text { Milan (Italie) } \\
\text { SE Wi (USA) } \\
\text { Sacramento CA (USA) } \\
\text { CA (USA) } \\
\text { Mendoza (Argentine) } \\
\text { Mendoza (Argentine) }\end{array}$ & $\begin{array}{l}\text { Conti GG } \\
\text { Walker JC } \\
\text { Hull R } \\
\text { Campbell RN } \\
\text { Pirone TP } \\
\text { Garcia O } \\
\text { Garcia O }\end{array}$ & $\begin{array}{l}\text { Lebeurier et al, } 1978 \\
\text { Walker et al, } 1945 \\
\text { Hull et Howell, } 1978 \\
\text { Shepherd et al, } 1968\left(^{*}\right) \\
\text { Shalla et al, } 1980 \\
\text { Garcia et Shepherd, } 1985 \\
\text { Garcia, comm personnelle }\end{array}$ \\
\hline
\end{tabular}

(" American Type Culture Collection, Catalogue of strains II, 1981, p 387). 


\section{Méthodes}

\section{Indexage}

L'indexage des plantes tests (4 par échantillon) a été réalisé par inoculation mécanique selon la technique classique (Kerlan et Mevel, 1989). L'inoculum a toujours été préparé à partir de $B$ campestris. L'incubation a eu lieu en serre dans les conditions précédemment décrites (Kerlan et Mevel, 1989).

\section{Contrôle de l'infection}

L'infection chez les plantes inoculées a été contrôlée, soit par rétro-inoculation sur Brassica campestris ou $B$ pekinensis (Lour) pour les feuilles présentant des lésions locales, soit par la technique ELISA pour les feuilles néoformées.

La technique ELISA (Clark et Adams, 1977) a été mise en œuvre avec des réactifs obtenus à partir d'un immunsérum préparé au laboratoire contre la souche Cabb-S et selon un protocole déjà mentionné pour la détection de ce virus à partir de chou-fleur (Kerlan et Mevel, 1987). Les densités optiques sont lues aux spectrophotomètre (Flow) à $405 \mathrm{~nm}$ après $2 \mathrm{~h}$ d'incubation enzyme-substrat, à température ambiante.

\section{Purification du virus}

Le virus a été purifié à partir de $B$ pekinensis après 4 semaines de multiplication en utilisant la technique de Hull et al (1976).

\section{Microscopie électronique}

Les observations en microscopie électronique ont été effectuées sur un appareil JEOL $100 \mathrm{CX}$ à l'Université de Rennes-l. Les préparations obtenues après mise en ceuvre de la "dip-method" (Brandes, 1957) ont été colorées à l'acétate d'uranyle à $2 \%$.

\section{RÉSULTATS}

Sur 12 espèces de plantes testées, 4 présentent des réactions locales et systémiques : $D$ stramonium, $N$ bigelowii, $N$ clevelandii et $N$ benthamiana. Une seule, $N$ debneyi, est immune. Les 7 autres n'extériorisent des symptômes que sur les feuilles inoculées; l'inoculation de retour des lésions locales sur $B$ campestris ou $B$ pekinensis se traduit par des symptômes de chloroses et, éventuellement de nécroses, typiques du CaMV, ce qui confirme leur origine virale.

\section{Plantes hôtes à réactions locales et systémiques}

\section{Datura stramonium (tableau II)}

L'ensemble des 18 souches ou isolats a été inoculé à $D$ stramonium. Une seule souche, CM4184 , ne provoque aucune réaction et sa présence n'est pas détectable par la technique ELISA; $D$ stramonium apparaît donc immune vis-à-vis de cette souche. Toutes les autres sources d'inoculum expérimentées induisent des lésions locales, et seules 9 d'entre elles provoquent une réponse systémique (tableau II).

Sur les feuilles inoculées par les 17 souches ou isolats autres que CM4-184, des lésions locales apparaissent environ $15 \mathrm{j}$ après l'inoculation (fig 1); elles deviennent ensuite nécrotiques, excepté pour l'isolat B-23. Dans le cas des isolats $\mathrm{B}-26$ et $\mathrm{B}-32$, le processus de nécrotisation est très rapide et la phase chlorotique quasiinexistante. Pour chaque souche ou isolat, les 4 plantes inoculées ont réagi de la même façon.

La réponse systémique est par contre moins homogène. Les premiers symptômes apparaissent sur les feuilles néoformées 5 semaines après l'inoculation par les 9 souches ou isolats suivants : B-12, B-20, B-21, B-22, B-23, B-30, Cabb-BJI, W 260, A 517; ce sont des taches chlorotiques ou/et des points nécrotiques. Les taches chlorotiques, induites par les isolats B12, B-21, B-23, B-30 d'abord diffuses et internervaires, laissent place au cours des semaines suivantes à un éclaircissement nervaire très dense, sans déformation des feuilles (fig 2). Les points nécrotiques ont $1-2 \mathrm{~mm}$ de diamètre sur les feuilles jeunes (fig 3), 3-4 mm sur les feuilles

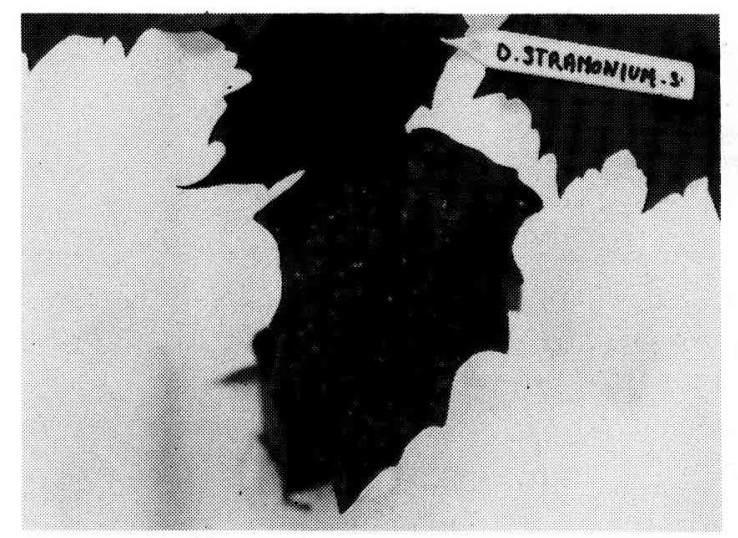

Fig 1. Lésions locales chlorotiques sur feuille de $D$ stramonium inoculée par la souche de référence Cabb-S, 15 j après inoculation. 
Tableau II. Réaction de Datura stramonium à l'infection par différents isolats et souches de CaMV.

\begin{tabular}{|c|c|c|c|c|c|c|c|c|}
\hline $\begin{array}{l}\text { Isolat ou } \\
\text { souche }\end{array}$ & $\begin{array}{l}\text { Réaction } \\
\text { locale }\end{array}$ & $\begin{array}{c}\text { Réaction } \\
\text { TC }\end{array}$ & $\begin{array}{c}\text { systémique sut } \\
\text { PN }\end{array}$ & $\begin{array}{c}\text { ur feuilles (a) } \\
D A\end{array}$ & $\begin{array}{c}\text { Symptômes } \\
\text { sur fruits }\end{array}$ & $\begin{array}{c}\text { Total d'inf } \\
\text { systémique (b) }\end{array}$ & $\begin{array}{l}\text { Densités optiques } \\
\text { plantes S }\end{array}$ & $\begin{array}{l}\text { SELISA (c) } \\
\text { plantes } \$\end{array}$ \\
\hline B-7 & $\mathrm{CN} \rightarrow \mathrm{N}$ & 0 & 0 & 0 & 0 & 0 & & 0,026 \\
\hline B-12 & $\mathrm{CN} \rightarrow \mathrm{N}$ & 3 & 1 & 0 & 0 & 4 & 0,378 & \\
\hline B-17 & $\mathrm{CN} \rightarrow \mathrm{N}$ & 0 & 0 & 0 & 0 & 0 & & 0,035 \\
\hline B-20 & $\mathrm{CN} \rightarrow \mathrm{N}$ & 0 & 2 & 2 & 2 & 2 & 0,360 & \\
\hline B-21 & $\mathrm{CN} \rightarrow \mathrm{N}$ & 2 & 2 & 0 & 0 & 4 & 1,251 & \\
\hline B-22 & $C \rightarrow N$ & 0 & 1 & 0 & 0 & 1 & 0,953 & 0,021 \\
\hline$B-23$ & C & 4 & 0 & 0 & 0 & 4 & 1,532 & \\
\hline B-26 & $\mathrm{N}$ & 0 & 0 & 0 & 0 & 0 & & 0,010 \\
\hline B-29 & $\mathrm{CN} \rightarrow \mathrm{N}$ & 0 & 0 & 0 & 0 & 0 & & 0,013 \\
\hline B-30 & $\mathrm{CN} \rightarrow \mathrm{N}$ & 1 & 0 & 1 & 1 & 1 & 1,122 & \\
\hline B-32 & $\mathbf{N}$ & 0 & 0 & 0 & 0 & 0 & & 0,032 \\
\hline Cabb-S & $\mathrm{CN} \rightarrow \mathrm{N}$ & 0 & 0 & 0 & 0 & 0 & & 0,030 \\
\hline Cabb-B & $\mathrm{C} \rightarrow \mathrm{CN}$ & 0 & 0 & 0 & 0 & 0 & & 0,025 \\
\hline Cabb-BJI & $\mathrm{CN} \rightarrow \mathrm{N}$ & 0 & 3 & 2 & 2 & 3 & 0,650 & \\
\hline PV-45 & $\mathrm{C} \rightarrow \mathrm{CN}$ & 0 & 0 & 0 & 0 & 0 & & 0,028 \\
\hline CM4-184 & $\$$ & 0 & 0 & 0 & 0 & 0 & & 0,029 \\
\hline W 260 & $\mathrm{C} \rightarrow \mathrm{CN}$ & 0 & 4 & 0 & 0 & 4 & 1,127 & \\
\hline A 517 & $\mathrm{CN} \rightarrow \mathrm{N}$ & 0 & 4 & 0 & 0 & 4 & 1,266 & \\
\hline
\end{tabular}

$\mathrm{N}$ : lésions locales nécrotiques; $\mathrm{C}$ : lésions locales chlorotiques; $\mathrm{CN}$ : lésions locales chloro-nécrotiques; $\mathrm{S}$ : avec symptômes; $\$$ : sans symptômes; TC : taches chlorotiques; PN : points nécrotiques; DA : dégénérescence apicale; a) : nombre de plantes, sur 4 testées, ayant présenté ce type de réaction; b) : total des plantes infectées systémiquement : taches chlorotiques, points nécrotiques, dégénérescence apicale; c) : test ELISA effectué sur les feuilles néoformées 10 semaines après l'inoculation : - les valeurs données correspondent à une moyenne lorsque plusieurs plantes sont infectées, - les valeurs obtenues pour 4 plantes-témoins non inoculées varient entre 0,010 et 0,030 .

âgées où ils demeurent généralement noncoalescents; ce dernier type de symptôme est le plus fréquent; sur les 36 plantes inoculées par les 9 souches ou isolats induisant une réaction systémique, 17 ont réagi de cette façon, 4 isolats - B-12, B-20, B-21, B-22 - et 3 souches - CabbBJl, W 260 et A 517 - étant concernés. B-12 et

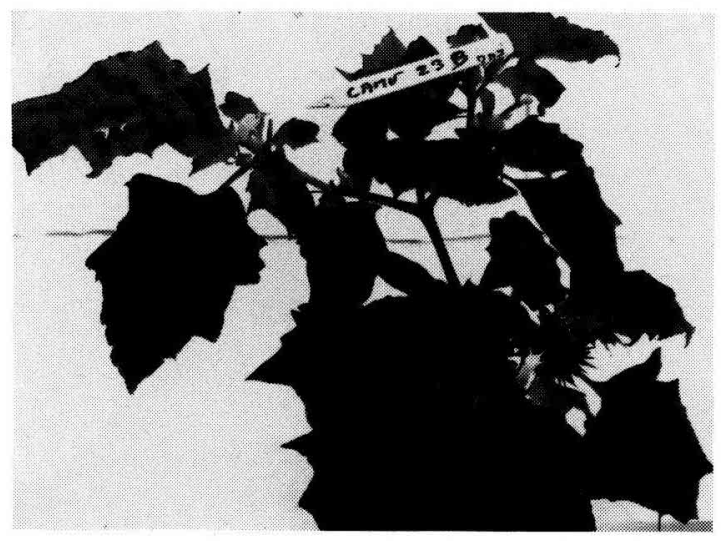

Fig 2. Réaction systémique de $D$ stramonium : réseau chlorotique, 6 semaines après l'inoculation par la souche B-23.
B-21 ont la particularité d'induire les 2 types de symptômes, taches chlorotiques sur certaines plantes, points nécrotiques sur les autres. Par contre l'inoculation des isolats B-23 et B-30 ne se traduit que par des réactions de type chlorotique.

A un stade plus avancé (8-10 semaines après l'inoculation), certaines plantes, ayant réagi par des taches chlorotiques ou des nécroses systé-

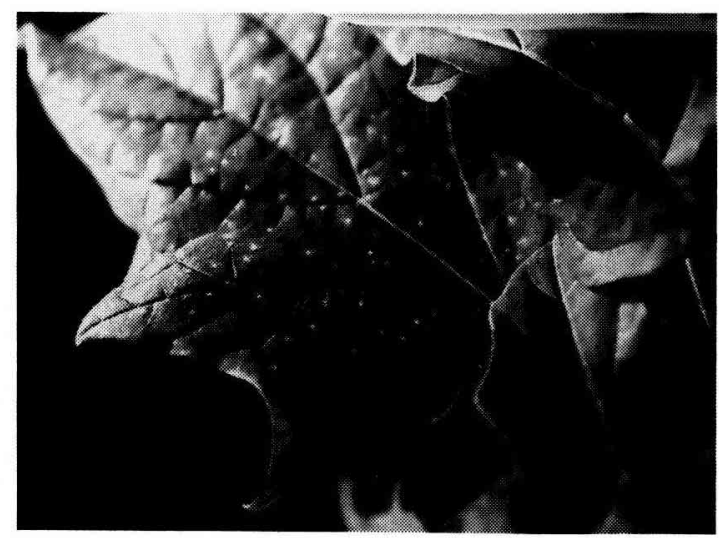

Fig 3. Réaction systémique de $D$ stramonium : points nécrotiques induits par la souche B-20, 6 semaines après l'inoculation. 
miques, présentent en outre une forte réaction de dégénérescence apicale (fig 4); les feuilles nouvellement formées sont alors fortement gaufrées, de taille réduite et avec des nécroses nervaires. Ce type de réaction est observé sur 5 plantes tests inoculées par les isolats B-20 (2/4) et B-30 (1/4) et la souche Cabb-BJI (2/4). La phase terminale consiste en un dépérissement total des plantes atteintes (fig 5), avec auparavant formation de fruits et de graines très perturbés; les fruits, souvent craquelés et de calibre réduit, présentent des nécroses internes et externes et ne parviennent pas à maturité; les graines formées sont petites et d'aspect anormal. B-20, B-30 et Cabb-BJI sont donc capables de provoquer - au moins sur certaines plantes tests - des réactions particulièrement sévères.

La gamme de symptômes observés sur $D$ stramonium peut être récapitulée de la façon suivante :

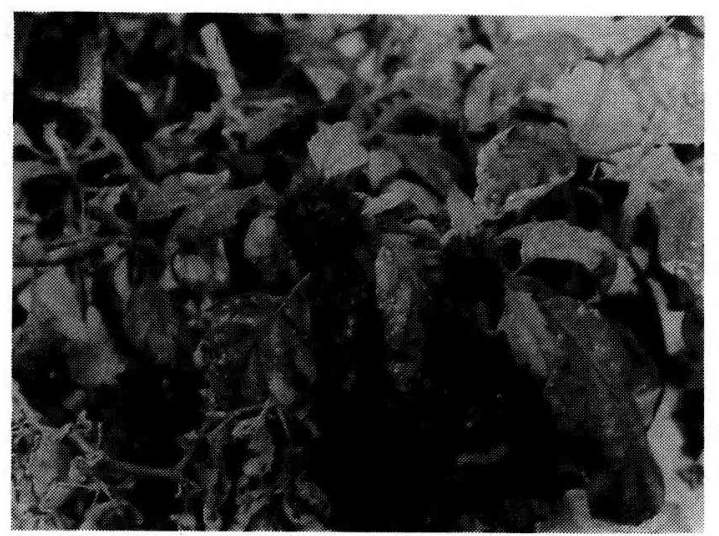

Fig 4. Réaction systémique généralisée de $D$ stramonium, 8 semaines après l'inoculation : points nécrotiques et début de dégénérescence apicale induits par la souche B-20.

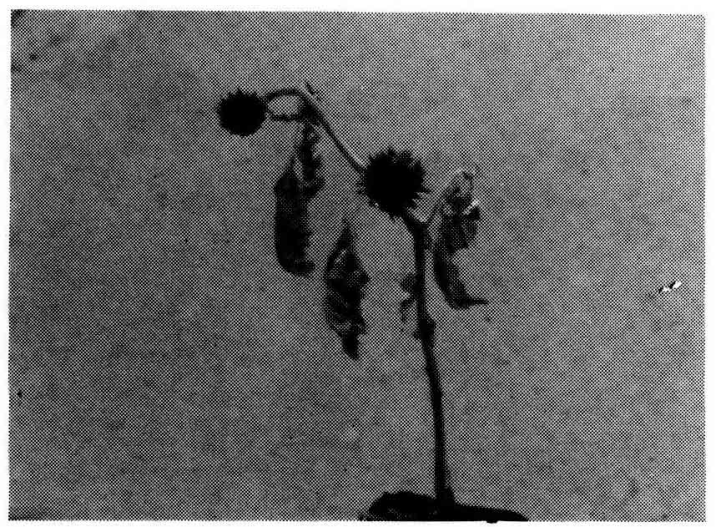

Fig 5. Dépérissement total de $D$ stramonium provoqué par la souche B-30, 10 semaines après l'inoculation; les fruits sont de taille réduite.
- immunité : une souche, CM4-184;

- réactions locales seulement : 5 isolats, B-7, B17, B-26, B-29, B-32 et 3 souches, Cabb-S, Cabb-B, PV-45;

- réactions locales et systémiques :

- pas de symptômes sur fruits : 4 isolats, $B$ 12, B-21, B-22, B-23 et 2 souches, W 260 et A 517 ;

- symptômes sur fruits : 2 isolats, B-20, B-30 et une souche, Cabb-BJl.

L'observation des symptômes a été contrôlée par un test ELISA réalisé sur un broyat de feuilles néoformées, 10 semaines après la date d'inoculation; les résultats confirment l'absence de virus chez les plantes ne présentant pas de symptômes systémiques. Les densités optiques positives sont variables, relativement fortes pour les isolats B-21, B-23, B-30 et les souches A 517 et W 260; l'intensité des densités optiques n'est pas corrélée avec un type de symptôme particulier, ni un taux faible ou élevé de plantes infectées.

\section{Nicotiana bigelowii (tableau III)}

Sur les 9 testés, 5 isolats ou souches, B-23, Cabb-S, Cabb-B, PV-45, et CM4-184, n'induisent aucune réaction, locale ou systémique. B$22, \mathrm{~B}-29, \mathrm{~B}-30$ et $\mathrm{W} 260$ provoquent la formation de lésions locales, le plus souvent nécrotiques, 2-3 semaines après l'inoculation. De plus, une réaction systémique se manifeste chez plusieurs plantes (respectivement $2 / 4$ et $3 / 4$ ) inoculées par B-29 et W 260; les symptômes qui consistent en des points, d'abord chlorotiques, puis nécrotiques, apparaissent sur les grandes feuilles néoformées 6-8 semaines après l'inoculation; plus tardivement, au stade préfloral, les jeunes feuilles de l'apex sont porteuses d'un réseau chlorotique internervaire.

Le test ELISA, effectué $42 \mathrm{j}$ après l'inoculation sur les feuilles néoformées, confirme la présence du virus chez les 5 plantes présentant des symptômes systémiques; toutes les autres valeurs de densités optiques ne sont pas significativement différentes de celles obtenues à partir de plantes saines.

\section{Nicotiana clevelandii (tableau III)}

$N$ clevelandii apparaît immune vis-à-vis des isolats B-23 et B-29 et des souches Cabb-S, CabbB, PV-45, CM4-184, qui n'induisent aucune réaction, locale ou systémique, l'absence de virus 
Tableau III. Réaction de Nicotiana bigelowii, $N$ clevelandi et $N$ benthamiana à l'infection par différentes souches de CaMV.

\begin{tabular}{|c|c|c|c|c|c|c|c|c|c|c|}
\hline $\begin{array}{c}\text { Isolat } \\
\text { ou souche }\end{array}$ & $\begin{array}{l}\text { Réaction } \\
\text { locale }\end{array}$ & $\begin{array}{l}\text { N bigelowii } \\
n \text { Réaction } \\
\text { systémique }\end{array}$ & $\begin{array}{c}D O \\
E L I S A(a)\end{array}$ & $\begin{array}{c}\text { Réaction } \\
\text { locale }\end{array}$ & $\begin{array}{c}\text { V clevelandii } \\
\text { Réaction } \\
\text { systémique }\end{array}$ & $\begin{array}{c}D O \\
E L I S A\end{array}$ & $\begin{array}{c}\text { N b } \\
\text { Réaction } \\
\text { locale }\end{array}$ & $\begin{array}{l}\text { Denthamiana } \\
\text { Réaction } \\
\text { systémique }\end{array}$ & & $\begin{array}{c}D O \\
E L I S A\end{array}$ \\
\hline B-22 & $\mathbf{N}$ & $\$$ & 0,023 & $\mathbf{N}$ & $\$$ & 0,015 & $\mathrm{C}$ & $E N+T C$ & 0,066 & 0,082 \\
\hline B-23 & $\$$ & $\$$ & 0,037 & $\$$ & $\$$ & 0,013 & $\$$ & $\$$ & 0,090 & 0,070 \\
\hline B-29 & $\mathrm{C}$ & $\begin{array}{c}2 / 4 \mathrm{PN} \\
2 / 4 \$\end{array}$ & $\begin{array}{l}0,296-0,233 \\
0,013-0,011\end{array}$ & $\$$ & $\$$ & 0,014 & $\mathrm{C}$ & $E N+T C$ & 0,074 & 0,049 \\
\hline B-30 & $N$ & $\$$ & 0,012 & $N$ & $\$$ & 0,023 & C & $E N+T C$ & 0,074 & 0,053 \\
\hline Cabb-S & $\$$ & $\$$ & 0,013 & $\$$ & $\$$ & 0,034 & $\$$ & $\$$ & 0,085 & 0,098 \\
\hline Cabb-B & $\$$ & $\$$ & 0,018 & $\$$ & $\$$ & 0,008 & $\$$ & $\$$ & 0,090 & 0,037 \\
\hline Cabb-BJI & & Non testée & & $\mathbf{N}$ & $\$$ & 0,010 & C & TC & 0,070 & 0,065 \\
\hline PV-45 & $\$$ & $\$$ & 0,014 & $\$$ & $\$$ & 0,011 & $\$$ & $\$$ & 0,086 & 0,070 \\
\hline CM4-184 & $\$$ & $\$$ & 0,008 & $\$$ & $\$$ & 0,020 & $\$$ & $\$$ & 0,060 & 0,084 \\
\hline W 260 & $\mathrm{~N}$ & $\begin{array}{c}3 / 4 P N \\
1 / 4 \$\end{array}$ & $\begin{array}{l}0,182 \\
0,018\end{array}$ & $N$ & TN & 0,196 & $\mathrm{C}$ & $E N+T C$ & 0,042 & 0,047 \\
\hline A 517 & & Non testée & & $N$ & $\begin{array}{l}3 / 4 \mathrm{TN} \\
1 / 4 \$\end{array}$ & $\begin{array}{l}0,184 \\
0,018\end{array}$ & C & $E N+T C$ & 0,023 & 0,063 \\
\hline
\end{tabular}

$\mathrm{N}$ : lésions locales nécrotiques; PN : points nécrotiques; $\mathrm{C}$ : lésions locales chlorotiques; TN : taches nécrotiques; $\$$ : pas de symptômes; TC : taches chlorotiques; EN : éclaircissement nervaire.

a) Test ELISA effectué sur les feuilles néoformées, 6 semaines ( $N$ bigelowii, $N$ clevelandii), 5 et 10 semaines ( $N$ benthamiana) après l'inoculation. Les résultats représentent la moyenne des D O pour 4 plantes tests excepté pour B-29 et W 260 chez $N$ bigelowii et $A$ 517 chez $N$ clevelandii; les valeurs obtenues pour 4 plantes témoins non inoculées varient entre 0,005 et 0,015 ( $N$ bigelowii), 0,007 et 0,023 ( $N$ clevelandii), 0,026 et 0,044 ( $N$ benthamiana).

étant confirmée par la technique ELISA et par inoculation sur $B$ pekinensis. Les 2 souches argentines, W 260 et A 517, de même que la souche Cabb-BJl et les isolats français B-22 et B-30, entraînent sur les feuilles inoculées la formation de lésions locales $10-15 \mathrm{j}$ après l'inoculation; d'abord chlorotiques, ces lésions se nécrosent très rapidement et ont un diamètre de 2-3 $\mathrm{mm}$.

Seules les 2 souches argentines induisent une réaction systémique sur $N$ clevelandii; ce sont des taches nécrotiques fortement marquées, de forme irrégulière, qui apparaissent brusquement au cours de la $5^{\mathrm{e}}$ semaine après l'inoculation; la technique ELISA effectuée à ce moment sur les feuilles présentant ce type de symptômes confirme la présence systémique du CaMV.

\section{Nicotiana benthamiana (tableau III)}

Sur les 11 expérimentés, 5 isolats ou souches, B-23, Cabb-S, Cabb-B, PV-45, CM4-184, n'induisent aucun symptôme, local ou systémique; les 6 autres, B-22, B-29, B-30, Cabb-BJI, W 260 et A 517, entraînent l'apparition de réactions lo- cales et systémiques. Dans tous les cas, les 4 plantes tests inoculées par le même isolat ou la même souche, réagissent de la même façon (fig 6 et 7).

Sur les feuilles inoculées, les lésions locales apparaissent $15 \mathrm{j}$ après l'inoculation; ce sont des taches chlorotiques de 2-3 $\mathrm{mm}$ de diamètre qui peuvent devenir ensuite coalescentes. Sur les autres feuilles, les premiers symptômes sont observables 5-6 semaines après l'inoculation; il

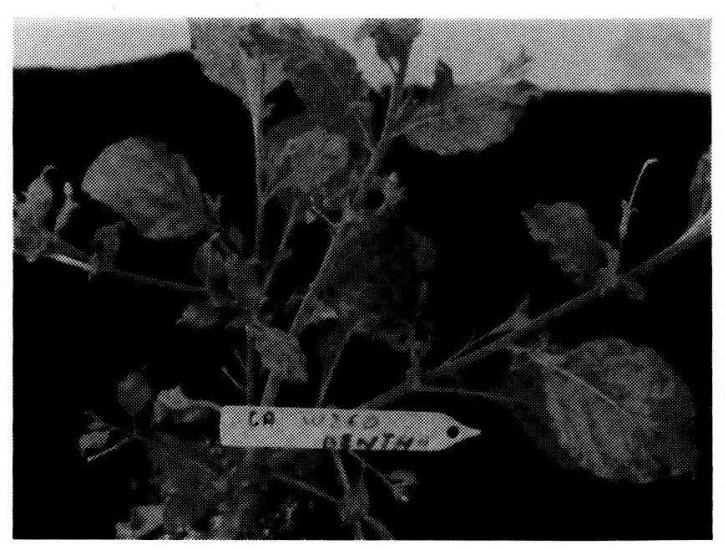

Fig 6. Taches chlorotiques systémiques sur $N$ benthamiana, 8 semaines après l'inoculation par la souche W 260. 


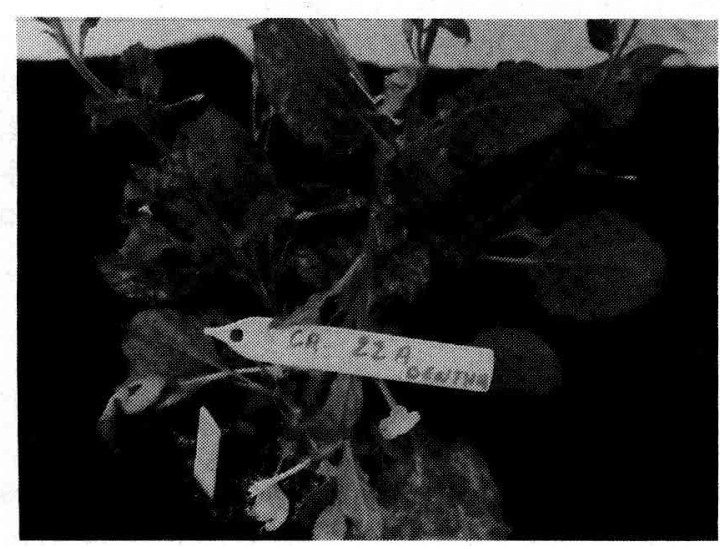

Fig 7. Taches chlorotiques systémiques sur $N$ benthamiana, 8 semaines après l'inoculation par la souche B-22.

s'agit de chloroses, soit limitées aux nervures (B22, B-29, W 260), soit accompagnées de taches internervaires (B-30, A 517); à ce moment, aucun symptôme n'est visible sur les plantes inoculées par la souche Cabb-BJI. A un stade ultérieur, les taches chlorotiques se généralisent et ont des diamètres variables : de $1 \mathrm{~mm}$ en moyenne Cabb-BJl) à 4-5 mm (B-22, B-29, W 260); dans le cas de l'isolat B-29, l'éclaircissement nervaire reste très fortement marqué. De façon générale, cette présence de symptômes foliaires s'accompagne d'un nanisme des plantes.

Deux séries de tests ELISA ont été effectuées pour confirmer l'infection systémique, 38 et $70 \mathrm{j}$ après l'inoculation sur les feuilles néoformées; les densités optiques obtenues sont très faibles, assez peu supérieures à celles des témoins sains; il n'apparaît pas de différences significa- tives entre plantes avec symptômes et plantes sans symptômes. Le test ELISA ne confirme donc pas la présence de virus chez cette plante.

Une inoculation de retour à partir des plantes extériorisant des réactions systémiques a alors été effectuée sur $B$ pekinensis qui a réagi normalement par des symptômes typiques de CaMV. Le virus a été purifié à partir de cette plante hôte inoculée par l'isolat B-22 et la souche W 260 . Le profil d'absorption spectrophotométrique est identique à celui généralement obtenu pour le CaMV. L'observation des suspensions purifiées en microscopie électronique révèle des particules morphologiquement identiques à celles habituellement décrites pour le CaMV. La technique ELISA appliquée aussi bien sur les extraits bruts de $B$ pekinensis que sur les suspensions purifiées confirme la présence du CaMV. En conclusion, ces 3 isolats, B22, B-29, B-30 et ces 3 souches, Cabb-BJI, W 260 et $A 517$, sont systémiques chez $N$ benthamiana.

\section{Plantes hôtes à réactions locales (tableau IV)}

Sept espèces ou variétés de plantes tests ne présentent des symptômes que sur les feuilles inoculées; ce sont : $N$ edwardsonii, $N$ glutinosa, $N$ glauca, $N$ tabacum Turkish, $N$ tabacum Samsum, $N$ tabacum Xanthi, $N$ rustica. Les résultats des tests ELISA et des rétro-inoculations sur $B$ campestris effectués à partir des feuilles néoformées des plantes inoculées sont identiques à ceux obtenus à partir de plantes saines; chez

Tableau IV. Réactions locales différenciatrices obtenues sur une gamme de Nicotiana inoculées par différents isolats et souches de CaMV.

\begin{tabular}{|c|c|c|c|c|c|c|c|}
\hline $\begin{array}{l}\text { Isolat ou } \\
\text { souche }\end{array}$ & $\mathrm{N}$ edwardsonii & $\mathrm{N}$ glutinosa & $\mathrm{N}$ glauca & $\begin{array}{c}\mathrm{N} \text { tabacum } \\
\text { Turkish }\end{array}$ & $\begin{array}{l}\mathrm{N} \text { tabacum } \\
\text { Samsum }\end{array}$ & $\begin{array}{c}\mathrm{N} \text { tabacum } \\
\text { Xanthi }\end{array}$ & N rustica $N$ debneyi \\
\hline
\end{tabular}

\begin{tabular}{|c|c|c|c|c|c|c|c|c|}
\hline B-22 & $\mathbf{N}$ & $\$$ & $\mathbf{N}$ & $\$$ & $\$$ & $\$$ & $\$$ & $\$$ \\
\hline B-23 & $\$$ & $\$$ & $\mathbf{N}$ & $\$$ & $\$$ & $\$$ & $\$$ & $\$$ \\
\hline B-29 & $\mathbf{N}$ & $\mathbf{N}$ & $\mathbf{N}$ & $\$$ & $\$$ & $\$$ & $\$$ & $\$$ \\
\hline B-30 & $\mathbf{N}$ & $\$$ & $\mathbf{N}$ & $\$$ & $\$$ & $\$$ & $\$$ & $\$$ \\
\hline Cabb-S & $\$$ & $\mathbf{N}$ & $\mathbf{N}$ & $\$$ & $\$$ & $\$$ & $\$$ & $\$$ \\
\hline Cabb-B & $\$$ & $\mathbf{N}$ & $\mathbf{N}$ & $\$$ & $\$$ & $\$$ & $\$$ & $\$$ \\
\hline Cabb-BII & $\mathbf{N}$ & $\mathbf{N}$ & $\$$ & $\mathbf{N}$ & $\$$ & $\$$ & $\$$ & $\$$ \\
\hline$P V-45$ & $\$$ & $\mathbf{N}$ & $\$$ & $\$$ & $\$$ & $\$$ & $\$$ & $\$$ \\
\hline CM4-184 & $\$$ & $\mathbf{N}$ & $\$$ & $\$$ & $\$$ & $\$$ & $\$$ & $\$$ \\
\hline W 260 & $\mathbf{N}$ & $\mathbf{N}$ & $\$$ & $\$$ & $\mathrm{C}$ & $\mathrm{C}$ & $\mathrm{C}$ & $\$$ \\
\hline A 517 & $\mathbf{N}$ & $\mathbf{N}$ & $\$$ & $\mathbf{N}$ & $\mathrm{C}$ & $C$ & $C$ & $\$$ \\
\hline
\end{tabular}


ces 7 plantes hôtes, il n'y a donc pas de généralisation du virus, mais, au moins dans nos conditions d'expérience, une infection qui reste localisée.

\section{Nicotiana edwardsonii}

Trois isolats B-22, B-29, B-30 et 3 souches Cabb-BJI, W 260, A 517, induisent sur les feuilles inoculées la formation de lésions locales nécrotiques de $3 \mathrm{~mm}$ environ de diamètre, 18-20 $\mathrm{j}$ après l'inoculation.

\section{Nicotiana glutinosa}

Cette espèce réagit localement avec toutes les souches étrangères et 1 seul des isolats français, B-29. Les lésions nécrotiques, de diamètre 4 à $5 \mathrm{~mm}$, assez peu nombreuses, se manifestent 5-7 $\mathrm{j}$ après l'inoculation, excepté avec la souche $W 260$ pour laquelle leur apparition est plus tardive ( $15 \mathrm{j}$ en moyenne).

\section{Nicotiana glauca}

Quatre isolats français B-22, B-23, B-29, B-30 et 2 souches de référence $\mathrm{Cabb}-\mathrm{S}$ et $\mathrm{Cabb}-\mathrm{B}$, induisent des lésions locales chloronécrotiques de diamètre 4 à $5 \mathrm{~mm}, 15-20 \mathrm{j}$ après la date d'inoculation (fig 8).

\section{Nicotiana tabacum L Turkish}

Cette plante hôte n'est sensible qu'à 2 souches, Cabb-BJl et A 517; c'est la seule qui permette de différencier les 2 souches argentines; les lésions locales nécrotiques, de $2 \mathrm{~mm}$ de diamètre sont observables $12 \mathrm{j}$ après l'inoculation.

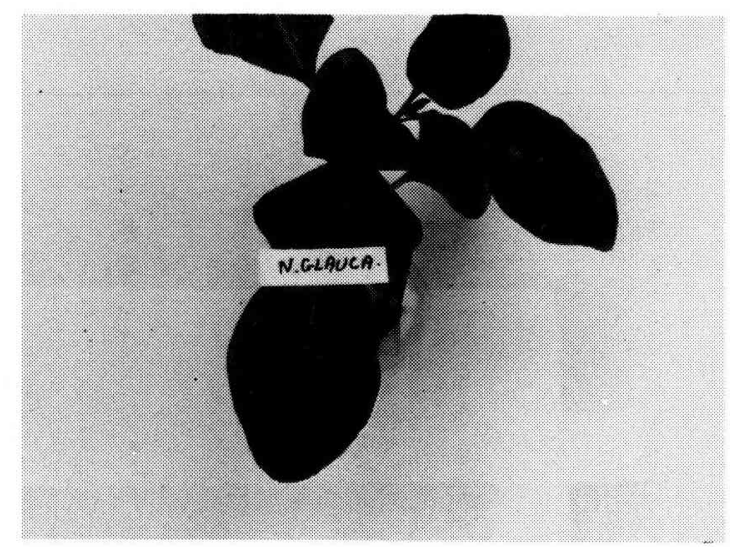

Fig 8. Lésions locales chloro-nécrotiques sur feuille inoculée de $N$ glauca par la souche B-29, $16 \mathrm{j}$ après l'inoculation.

\section{Nicotiana tabacum L Samsum, $N$ tabacum L Xanthi, N rustica}

Ces 3 plantes hôtes réagissent de la même façon; seules les 2 souches argentines y induisent des lésions locales chlorotiques de $3 \mathrm{~mm}$ de diamètre, 15-16 j après l'inoculation.

\section{Espèce immune : Nicotiana debneyi}

Aucun des 11 isolats ou souches expérimentés ne s'est révélé capable d'infecter localement ou systémiquement $N$ debneyi.

\section{DISCUSSION}

La présente étude montre que certains des isolats de CaMV obtenus à partir de prélèvements effectués sur chou-fleur en culture peuvent être systémiques sur Solanées, en l'occurence 4 espèces, $D$ stramonium, $N$ bigelowii, $N$ clevelandii et $N$ benthamiana.

$N$ benthamiana est la $6^{\circ}$ espèce de Solanée capable de réagir de façon systémique à certains isolats de CaMV, après $D$ stramonium, $N$ clevelandii (Gracia et Shepherd, 1985), $N$ edwardsonii, $N$ bigelowii et $N$ glutinosa (Schoelz et al, 1986). Dans nos conditions d'expérience, cette espèce présente une réaction très particulière caractérisée par une symptomatologie bien définie, mais non corrélée avec les résultats du test ELISA quant à la présence du virus dans la plante; les rétro-inoculations sur Crucifères confirment cependant la réalité de l'infection systémique. L'hypothèse la plus vraisemblable consiste en un taux de multiplication très faible du CaMV chez $N$ benthamiana.

Les réactions observées sur l'appareil végétatif de $D$ stramonium sont peu différentes de celles décrites par Schoelz et Shepherd (1988), mais ces auteurs ne font mention que des perturbations foliaires; il convient également de prendre en compte les symptômes sur fruits et graines qui sont spécifiques à certaines souches virales. Cependant, nous n'avons pas noté chez cette espèce de différences notables concernant les résultats du test ELISA, nous permettant de distinguer 2 types de systémie, totale et limitée, ainsi que le font Schoelz et Shepherd (1988); les valeurs de densité optique obtenues sont de façon générale plus élevées que celles mentionnées par ces auteurs. 
Tableau V. Infection systémique de quelques Solanées par différents groupes d'isolats et de souches du CaMV.

D stramonium $\mathrm{N}$ bigelowii $\mathrm{N}$ clevelandii $\mathrm{N}$ benthamiana $\mathrm{N}$ edwardsonii $\mathrm{N}$ glutinosa

\begin{tabular}{|c|c|c|c|c|c|c|}
\hline $\begin{array}{l}\text { Isolats français } \\
\text { Souches de référence }\end{array}$ & \pm & \pm & - & \pm & - & - \\
\hline Cabb-BJI & + & non testée & - & + & - & - \\
\hline autres & - & - & - & - & - & - \\
\hline Souches argentines & + & $+(a)$ & + & + & - & - \\
\hline Souche D4 (b) & + & + & + & - & + & + \\
\hline
\end{tabular}

+ : infection systémique; - : pas d'infection systémique; (a) : seule la souche W 260 a été testée sur $N$ bigelowi;; (b) : d'après Schoelz et Shepherd (1986).

La souche Cabb-BJl se différencie nettement de la souche Cabb-B dont elle dérive et des 3 autres souches de référence, en particulier par son aptitude à infecter systémiquement $D$ stramonium et $N$ benthamiana. II faut cependant noter que toutes les 5 sont capables d'infecter au moins une Solanée, ainsi qu'en témoigne la réaction d'hypersensibilité sur $\mathbf{N}$ glutinosa; de plus, seule CM4-184 ne provoque pas de réaction locale sur $D$ stramonium.

Plusieurs isolats français, ainsi que les 2 souches argentines sont dans l'ensemble assez proches de Cabb-BJI (tableau V); ils diffèrent assez nettement de la souche américaine D4, celle-ci étant systémique sur $N$ clevelandii, $N$ edwardsonii et $N$ glutinosa alors qu'elle n'infecte pas $\mathbf{N}$ benthamiana (Schoelz et al, 1986).

Les 2 souches argentines se distinguent des isolats français par leur aptitude à infecter de façon systémique $\mathbf{N}$ clevelandii. Dans nos conditions d'expérience, nous confirmons pour W 260, les résultats rapportés par Garcia et Shepherd (1985) sur $N$ clevelandii, ainsi que ceux de Schoelz et Shepherd (1988) sur $N$ edwardsonii (infection non systémique), $N$ bigelowii et $D$ stramonium; il convient cependant de noter la différence importante entre ces auteurs et nous au niveau de la détection immuno-enzymatique de $W$ 260 chez cette dernière espèce ( $D O=0,055$ contre 1,127).

En matière de classification, les souches de CaMV décrites à ce jour peuvent être globablement réparties de la façon suivante :

- les souches strictement inféodées aux Crucifères, telles celles décrites en Europe de l'Est par Horvath et al (1980);
- les souches capables d'infecter des Solanées avec 3 sous-groupes selon qu'elles sont, fortement, faiblement ou non systémiques, chez 3 espèces, $D$ stramonium, $N$ edwardsonii et $N$ bigelowii (Schoelz et Shepherd, 1988).

Aucun de nos isolats ne s'est révélé systémique sur $N$ edwardsonii, un seul sur $N$ bigelowii; nous n'avons pas observé chez $D$ stramonium de ségrégation entre infection forte et infection limitée. Cependant, cette dernière espèce, en raison du large spectre de ses réactions et de sa sensibilité à un grand nombre de souches et d'isolats ( 17 sur 18 testés), nous a semblé particulièrement interessante en tant que hôte différenciateur; elle nous semble constituer un bon critère de séparation des souches virales au même titre que $\boldsymbol{N}$ clevelandii et $B$ campestris qui nous avaient déjà permis d'établir une première distinction entre isolats français (Kerlan et Mevel, 1989).

En tenant compte de ces nouveaux aspects de la variabilité naturelle du CaMV, une prospection élargie à d'autres zones géographiques que la Bretagne et à d'autres Crucifères, cultivées ou non, devrait permettre de découvrir encore des isolats originaux de ce virus. Dans l'immédiat, l'étude de la capacité des isolats français a être transmise par diverses espèces d'Aphides est en cours pour compléter leur caractérisation au plan biologique.

\section{REMERCIEMENTS}

Nous avons le plaisir de remercier f'Institut français du tabac de Bergerac qui nous a fourni les graines des différentes espèces de Nicotiana utilisées dans cette étude. 


\section{RÉFÉRENCES}

Brandes J (1957) Eine elektronenmikroskopische Schnellmethode zum Nachweis Faden und Sträbchenförmiger Viren insbesondere in Kartoffeldunkerdeimen. Nachrichtenbl Deutsch Pflanzenschutzdienstes Braunschw 9, 151-152

Clark MF, Adams AN (1977) Characteristics of the microplate method of enzyme-linked immunosorbent assay for the detection of plant viruses. $J$ Gen Virol 34, 475-483

Garcia O, Shepherd RJ (1985) Cauliflower mosaic virus in the nucleus of Nicotiana. Virology 146, 141145

Hills GJ, Campbell RN (1968) Morphology of broccoli necrotic yellows virus. J Ultrastruct Res 24, 134144

Horvath J, Besada WH, Juretic N, Mamula D (1980) Some data on properties of cauliflower mosaic virus in Hungary. Tag Ber Akad Landwirtsch Wiss $D D R$, Berlin 184, 53-60

Hull R, Shepherd RJ, Harvey JD (1976) Cauliflower mosaic virus: an improved purification procedure and some properties of the virus particles. $J$ Gen Virol 31, 93-100

Hull R, Howell SH (1978) Structure of the cauliflower mosaic virus genome II. Variation in DNA structure and sequence between isolates. Virology $86,482-$ 493
Kerlan C, Mevel S (1987) Practical use of ELISA to detect cauliflower mosaic virus in Cauliflower. Eur Mediterr Plant Prot Organ 17, 125-130 (EPPO Bull)

Kerlan C, Mevel S (1989) Variabilité biologique du virus de la Mosaïque du chou-fleur sur chou-fleur en Bretagne. Agronomie 9, 83-90

Lebeurier G, Whitechurch O, Lesot A, Hirth L (1978) Physical map of DNA from a new cauliflower mosaic infection. Virology 37, 506-508

Lung MCY, Pirone TP (1972) Datura stramonium, a local lesion host for certain isolates of cauliflower mosaic virus. Phytopathology 61, 1473-1474

Schoelz JE, Shepherd RJ, Richins RD (1986) Properties of an unusual strain of cauliflower mosaic virus. Phytopathology 76, 451-454

Schoelz JE, Shepherd RJ (1988) Host range control of cauliflower mosaic virus. Virology 162, 30-37

Shalla TA, Shepherd RJ, Peterson LJ (1980) Comparative cytology of nine isolates of cauliflower mosaic virus. Virology 102, 381-388

Shepherd RJ (1981) Cauliflower mosaic virus. CMV/ AAB. Desc Plant Virus 243, 1-6

Shepherd RJ, Wakeman RJ, Romanko RR (1968) DNA in cauliflower mosaic virus. Virology 36,150 152

Walker JC, Lebeau FJ, Pound GS (1945) Viruses associated with cabbage mosaic. J Agric Res 70, 379404 